\title{
Non-invasive assessment of urinary bladder compliance using ultrasound: first validation study based on clinical urodynamic study
}

\author{
Xinyu Zhang ${ }^{1,2}$, Douglas A. Husmann ${ }^{3}$, Lance A. Mynderse ${ }^{3}$, Azra Alizad $^{4} \wedge$, Mostafa Fatemi $^{2} \wedge$ \\ ${ }^{1}$ School of Biomedical Engineering, Health Science Center, Shenzhen University, Shenzhen, China; ${ }^{2}$ Department of Physiology and Biomedical \\ Engineering, Mayo Clinic College of Medicine \& Science, Rochester, MN, USA; ${ }^{3}$ Department of Urology, Mayo Clinic College of Medicine \& \\ Science, Rochester, MN, USA; ${ }^{4}$ Department of Radiology, Mayo Clinic College of Medicine \& Science, Rochester, MN, USA \\ Contributions: (I) Conception and design: M Fatemi, A Alizad; (II) Administrative support: M Fatemi, DA Husmann; (III) Provision of study materials \\ or patients: M Fatemi, A Alizad, DA Husmann, LA Mynderse; (IV) Collection and assembly of data: M Fatemi, A Alizad, X Zhang; (V) Data analysis \\ and interpretation: X Zhang; (VI) Manuscript writing: All authors; (II) Final approval of manuscript: All authors. \\ Correspondence to: Mostafa Fatemi. Department of Physiology and Biomedical Engineering, Mayo Clinic College of Medicine \& Science, 200 1st. St. \\ SW, Rochester, MN, USA. Email: fatemi.mostafa@mayo.edu.
}

Background: This study aims to validate a recently introduced non-invasive method, ultrasound bladder vibrometry (UBV), for the assessment of detrusor compliance in patients with neurogenic bladders.

Methods: The study was carried out on 79 adult patients with neurogenic bladders (60 male and 19 female). The UBV test was performed on each patient to measure the Lamb wave group velocity $\left(c_{g}\right)$ in the anterior bladder wall at every $50 \mathrm{~mL}$ volume increment throughout the filling phase. Bladder compliance was assessed based on the trend of Lamb wave group velocity squared $\left(c_{g}{ }^{2}\right)$ versus volume. A compliance index was defined to differentiate between the compliant and non-compliant bladders. Results of the UBV compliance assessment were validated using the readings of the corresponding urodynamic studies as the clinical gold standard.

Results: The Patients' bladders were divided into non-compliant and compliant groups by an experienced urologist using the information in the urodynamic study (UDS) recordings. The compliance index defined on the basis of $c_{g}{ }^{2}$ showed a significant difference $(\mathrm{P}<0.008)$ between the compliant and non-compliant groups. The areas under the receiver operating characteristic curve were 0.813 , with $95 \%$ CI ranging from 0.709 to 0.892 . Under the optimal criterion, the bladder was considered as non-compliant if the compliance index was less than $100 \mathrm{~mL} \cdot \mathrm{s}^{2} / \mathrm{m}^{2}$, resulting in a sensitivity and specificity of $86.4 \%$ and $71.9 \%$, respectively.

Conclusions: The results of this study demonstrate that UBV can be used as a non-invasive method for the determination of bladder compliance; thus, it can potentially serve as an alternative method to UDS for the appropriate patient groups.

Keywords: Bladder; bladder compliance; detrusor pressure; Lamb wave; ultrasound; urodynamic study (UDS)

Submitted Oct 15, 2020. Accepted for publication Jan 17, 2021.

doi: $10.21037 /$ atm-20-6900

View this article at: http://dx.doi.org/10.21037/atm-20-6900

^ ORCID: Azra Alizad, 0000-0002-7658-1572; Mostafa Fatemi, 0000-0002-6603-9077. 


\section{Introduction}

Proper bladder function is essential for the creation of urinary continence, long-term kidney health, and overall quality of life. Urodynamics (UDS) refers to an invasive diagnostic test that can provide objective pathophysiological explanations for symptoms and/or dysfunction of the lower urinary tracts. Filling cystometry, also known as a cystometrogram (CMG), is an important component of UDS and measures the pressure/volume relationship of the bladder throughout bladder filling. It is used to assess detrusor activity and bladder sensation, capacity, and compliance (1). CMG can be performed with a single urethral catheter alone for bladder filling and measurement of the intravesical pressure $\left(\mathrm{P}_{\mathrm{ves}}\right)$ or with an additional catheter that simultaneously measures intra-abdominal pressure $\left(\mathrm{P}_{\mathrm{abd}}\right)$ through the rectum or vagina. The multi-channel test has advantages in measuring the net detrusor pressure $\mathrm{P}_{\text {det }}$ by subtracting $\mathrm{P}_{\mathrm{abd}}$ (or extrinsic forces) from $\mathrm{P}_{\text {ves }}$ (i.e., $\left.P_{\text {det }}=P_{v e s}-P_{a b d}\right)$. The $\mathrm{P}_{\text {det }}$ refers to the component of $\mathrm{P}_{\mathrm{ves}}$ that is created by forces in the bladder wall (1) and is representative of the detrusor muscle of the bladder. CMG is performed in either seated, standing, or supine positions while the bladder is filled typically at a set rate of $25 \mathrm{~mL} / \mathrm{min}$. In general, the filling rate influences the accuracy of measurements. Studies by Kim et al. (2) have shown that slower filling rate would result in a more reliable estimation of the bladder compliance. The assessment of bladder compliance is one of the major functions of a complete urodynamic study. Normally, the bladder is very compliant and can accommodate large volumes of urine with very little increase in $\mathrm{P}_{\text {det }}$. Bladder compliance is calculated by dividing the volume change by the change in detrusor pressure. Normal bladder compliance is defined as a value of $>40 \mathrm{~mL} / \mathrm{cmH}_{2} \mathrm{O}$, with values of $<13 \mathrm{~mL} / \mathrm{cmH}_{2} \mathrm{O}$ representing severe compromise of bladder compliance (3). Assessment of detrusor compliance in patients with a neuropathic bladder is critical with individuals with low or poor detrusor compliance being at high risk for upper tract deterioration and induced renal failure (4). It is recommended that at-risk patients be carefully monitored with urodynamic studies to ensure the bladder pressure stay within a normal range thereby reducing the risk of renal damage.

Urodynamic tests represent the gold standard for measuring bladder compliance, but are invasive, uncomfortable and carry risks of iatrogenically caused urinary tract infections. These procedures last approximately 45 minutes or more, require catheter placement in the bladder and the vagina or rectum, filling the bladder at a defined rate, and measuring pressures throughout filling. Approximately half of the patients undergoing these studies have perceptions of either physical or emotional discomfort related to the performance of this test (5). Urinary catheter in and of itself may be a traumatic event that places the patient at risk for the induction of bacteria with significant bacteriuria rates following catheterization ranging from $1.50 \%$ to $14.0 \%$ based on specific sub-populations demographics $(6,7)$. While rare, the placement of a urinary catheter can cause trauma that may result in significant bleeding and urinary retention, requiring prolonged catheterization for treatment. There is no doubt that noninvasive measurement of bladder compliance and detrusor activity could be instrumental in clinical practice and could avoid the risk posed by current studies.

In this regard, ultrasound-based technology shows great potential for allowing us to obtain information on detrusor function in a non-invasive fashion. Nagle et al. demonstrate the quantification of several new biomechanical properties of the bladder, including wall tension, wall strain, wall stress, and dynamic elasticity, by the combination of ultrasound images data and $\mathrm{P}_{\text {ves }}(8)$. Later, this group demonstrated that an accurate and precise measurement of bladder volume could be realized using a $3 \mathrm{D}$ convex transducer and automatically reconstruction of the rendered volume, which may provide an important tool for non-invasive UDS (9).

Ultrasound shearwave elastography (USE) has emerged as non-invasive tool for assessment of the tissue mechanical properties in recent years (10). USE refers to a category of the quantitative techniques in which shear waves are generated in tissues by the excitation of a mechanical vibrating device or acoustic radiation force. Ultrasound techniques are used to measure the propagation speed of shear waves, which is related to Young's modulus E as $E=3 \rho c_{g}{ }^{2}$, where $c_{g}$ is the group velocity of the shear wave and $\rho$ is the density of the tissue. The USE test reported $c_{g}$ or the converted Young's modulus E to provide a quantitative indicator of tissue elasticity. In a thin-plate structure, vibrational waves directly interact with the surface of the structure, resulting in so-called Lamb waves. The propagation of Lamb waves depends on the density, the thickness and the elastic properties of a plate. The vibrational waves inside the bladder wall are treated as Lamb waves in a method called ultrasound bladder vibrometry (UBV) (11-13).

The detrusor pressure that is measured with urodynamic studies results from the combination of the tone of the detrusor muscle and the intrinsic viscoelasticity properties 
of the bladder wall; both components affect the mechanical properties of the bladder wall. In UBV, it is possible to monitor the response of the detrusor muscle non-invasively without the need for catheterization. Our group has conducted extensive studies on the application of UBV (11-14). These studies reveal that both Lamb wave speed (LWS) and the elasticity estimated based on the Lamb wave model show a strong correlation with $\mathrm{P}_{\text {det }}$ in healthy subjects (11) and adult patients (12). Some other groups treated the vibrational waves in the bladder wall as shear waves measured by a clinical elasticity imaging system and studied the correlation between the wave speed and bladder compliance (15). However, it should be noted that the shear wave assumption may not be valid due to the relatively small thickness of the bladder wall compared to the shear wave wavelength. For this reason, a Lamb wave model would be more appropriate for such waves in the bladder wall.

Building upon our previous studies, here we investigate, for the first time, the performance of UBV as a diagnostic tool for the differentiation between compliant and noncompliant bladders in a group of patients with neurogenic bladders. In particular, we defined a new parameter, called the compliance index, and evaluate its ability to discriminate between the non-compliant and compliant bladders. We validate the performance of the UBV based on independent clinical diagnosis of bladder compliance as the ground truth. We present the following article in accordance with the STARD reporting checklist (available at http://dx.doi. org/10.21037/atm-20-6900).

\section{Methods}

\section{Study population}

Adult patients with neurogenic bladder who were referred to the Mayo Clinic urology department for routine UDS were considered for the study. Exclusion criteria included dysreflexia, obese patients, patients with a history of prolonged catheter drainage, previous pelvic radiation/ surgery and pregnant or breastfeeding women. The study was conducted in accordance with the Declaration of Helsinki (as revised in 2013). This prospective study was Health Insurance Portability and Accountability Act Compliant and it was approved by the Mayo Clinic institutional review board (IRB\#: 11-001953), and written consent was obtained from patients prior to the examination. From March 2013 to January 2018, a total of 79 consecutive patients (60 male and 19 female) were recruited for this study.

\section{Experimental design-concurrent UDS and UBV}

Concurrent UDS and UBV were conducted on a group of patients. The UDS was performed and reported in accordance with the standards of the International Continence Society (ICS) (1). First, the bladder was catheterized through the urethra for gradual filling and simultaneous measurement of $\mathrm{P}_{\text {ves }}$ by a pressure sensor. An additional sensor is used to measure the pressure outside the bladder (i.e., $\mathrm{P}_{\text {abd }}$ ) via a rectal or vaginal catheter. The net $\mathrm{P}_{\text {det }}$ on the bladder wall is defined as the difference between the two pressure values. Pump volume, filling rate, pressure readings, and other parameters were recorded in a standard UDS chart. An experimental UBV system was implemented on a Verasonics ${ }^{\mathrm{TM}} \mathrm{V} 1$ research ultrasound system (Verasonics, Redmond, WA, USA) equipped with a curved linear array (C4-2, ATL/Philips, Bothell, WA, USA) with a working frequency of $2.5 \mathrm{MHz}$. The bladder was located in the B-mode image. Prior to each study, the patient's bladder was emptied, and pressure sensors were placed and calibrated to ensure consistent readings. Following standard clinical procedure guidelines (1), the clinical staff incrementally filled the bladder via the urethral catheter. For all of these studies a constant filling rate of $25 \mathrm{~mL} / \mathrm{min}$ was used. Two or three UBV acquisitions were performed by a sonographer at every increment of filling. Along with each acquisition, a timestamp was placed on the UDS data chart to locate the simultaneous pressure reading. For the UBV measurements, as shown in Figure 1, an ultrasound tone burst of 600-900 $\mu$ s was applied to the focal point on the anterior wall of the bladder at each acquisition. The resulting acoustic radiation force generated Lamb waves traveling along the bladder wall. These waves were then tracked at 2,500 frames per second using ultrasound plainwave ultrafast imaging with three-angle compounding (16). Particle displacement along the bladder wall was calculated from the recorded in-phase and quadrature (IQ) data using the autocorrelation technique in (17). The group velocity $c_{g}$ of Lamb waves was then calculated according to spatiotemporal maps of the wave propagation. The values of $c_{g}$ measured in two or three acquisitions were averaged and recorded at each increment of filling volume. The corresponding pressure readings of $\mathrm{P}_{\text {det }}$ and $\mathrm{P}_{\text {ves }}$ were also recorded by the UDS system.

\section{Definition of bladder compliance}




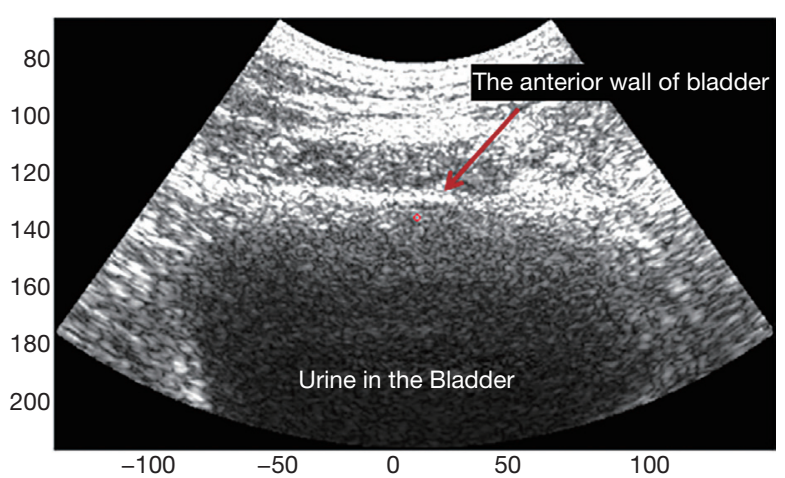

Figure 1 The anterior wall of bladder as seen in the B-mode ultrasound image, with a red circle showing the selected focal point on the bladder wall for ultrasound bladder vibrometry (UBV) measurement.

Bladder compliance, which describes the relationship between change in bladder volume and change in detrusor pressure, is generally regarded as a measure of bladder distensibility and is the key determinant regarding the patient's risk for upper urinary tract deterioration (3). The normal range for bladder compliance in adults has not yet been validated, and previous reports suggested the lowest value of compliance that should be accepted as normal as ranging from $12.5-40.0 \mathrm{~mL} / \mathrm{cmH}_{2} \mathrm{O}$ (18). Here, in this study, an experienced urologist reviewed the UDS recordings of the patient and calculated the compliance as follows:

$$
\text { Compliance }=\frac{\Delta V(m L)}{\Delta P\left(\mathrm{cmH}_{2} \mathrm{O}\right)}
$$

Here, $\Delta P$ is the difference between the detrusor pressure at the start of bladder filling at the corresponding bladder volume (usually zero) and the detrusor pressure corresponding to the bladder volume at cystometeric capacity or immediately before the start of any detrusor contraction that causes significant leakage, and $\Delta V$ is the difference between the bladder volumes at the start and at the end of filling.

Bladders with compliance of less than $40.0 \mathrm{~mL} / \mathrm{cmH}_{2} \mathrm{O}$ were considered as non-compliant bladders. For the UBV method, we defined a new LWS-related parameter, as follows, and proposed it as an indicator of bladder compliance:

Compliance Index $=\frac{\operatorname{Volume}(m L)}{c_{g}{ }^{2}\left(m^{2} \cdot s^{-2}\right)} @$ Last acquisition,

Where $c_{g}{ }^{2}$ and the volume are the group velocity square and the corresponding filling volume at the last UBV acquisition, respectively. Note that end-volume is close to but different from maximum capacity. The UBV acquisitions were made at about each $50 \mathrm{~mL}$ volume increment, but no acquisition was made at the last moment when the patient could not hold the urine any longer, and the urologist allows the patient to void.

\section{Statistical analyses}

Here we propose the group speed squared, $c_{g}^{2}$, as an indicator of bladder mechanical properties. Figure 2 shows the changes in $c_{g}{ }^{2}, \mathrm{P}_{\text {det }}$ and $\mathrm{P}_{\text {ves }}$ versus the filling volume for typical cases of compliant and non-compliant bladders. For a compliant bladder, both $c_{g}{ }^{2}$ and the pressure readings show little changes until the filling volume reaches around $400 \mathrm{~mL}$, whereas they both start to increase remarkably at the low filling volume of $250 \mathrm{~mL}$ for a non-compliant bladder. The correlations of $c_{g}{ }^{2} v s . \mathrm{P}_{\text {det }}$ and $c_{g}{ }^{2} v s$. $\mathrm{P}_{\text {ves }}$ were evaluated using Pearson correlation. The overall correlations of $c_{g}{ }^{2} v s . \mathrm{P}_{\text {det }}$ and $c_{g}{ }^{2} v s$. $\mathrm{P}_{\text {ves }}$ were also evaluated using the aggregate measurements of all patients. An experienced urologist reviewed all patients' UDS reports for bladder compliance and divided them into two groups with compliant and non-compliant bladders. The urologist's diagnosis was used as the ground truth in our study. The compliance index, as defined in Eqn. [2], was used to evaluate bladder compliance based on the UBV method. The performance of this parameter was evaluated via the receiver operating characteristic (ROC) curve method. All statistical analyses were performed using OriginPro 2018C (OriginLab Corporation, Northampton, MA, USA).

\section{Results}

The study group included 79 patients (60 male; 19 female). Patients' ages ranged from 21 to 82 years, with the mean age of $51.2 \pm 17.2$ years. The body mass index (BMI) ranged from 17.3 to 34.2 , with a mean BMI of $26.3 \pm 4.60$. Table 1 presents the demographic data and the clinical characteristics of all study patients. The maximum capacity, also called cystometric volume, varied from patient to patient and ranged from 107 to $742 \mathrm{~mL}$ with the mean volume of $415 \pm 128 \mathrm{~mL}$. The filling phase ended at greater than $500 \mathrm{~mL}$ for 30 patients $(38.0 \%)$, and 10 patients (12.7\%) could not hold more than $250 \mathrm{~mL}$ due to the early leakage. No adverse events from performing the UBV or UDS noted. 

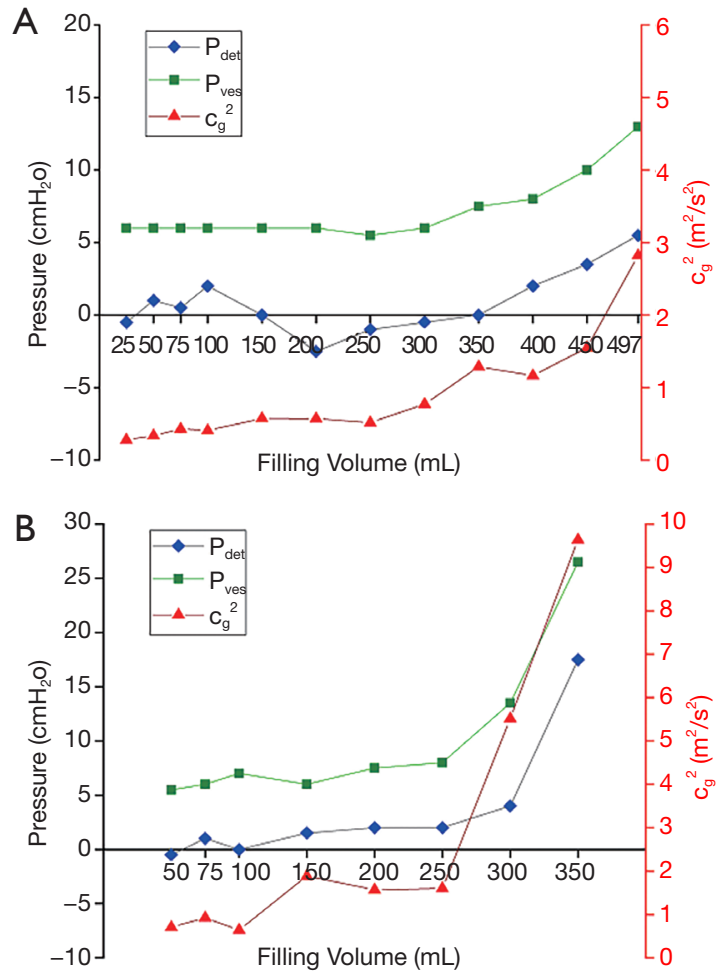

Figure 2 The changes of $\mathrm{P}_{\text {det }}, \mathrm{P}_{\text {ves }}$ and $c_{g}{ }^{2}$ versus the filling volume: (A) a typical case of a compliant bladder, and (B) a non-compliant bladder.

Table 1 The demographic data and clinical characteristics of all study patients

\begin{tabular}{lcc}
\hline Variables & Mean \pm STDEV & Range \\
\hline Age $(\mathrm{yrs})$ & $51.2 \pm 17.2$ & $21-82$ \\
BMI & $26.3 \pm 4.6$ & $17.3-34.2$ \\
Max capacity $(\mathrm{mL})$ & $415 \pm 128$ & $107-742$ \\
\hline
\end{tabular}

STDEV, standard deviation; Yrs, years; BMI, body mass index; Max, maximum; $\mathrm{mL}$, milliliter.

Before presenting the diagnostic performance of UBV, it is instructive to study the correlations between the UBV measured parameter, $c_{g}^{2}$, with the two key physiological pressures, $\mathrm{P}_{\text {det }}$ and $\mathrm{P}_{\text {ves }}$. Table 2 presents the correlation between $c_{g}{ }^{2}$ and $\mathrm{P}_{\text {det }}$ and $\mathrm{P}_{\text {ves }}$ for the 79 patients. The median and mean value of correlation coefficients between $c_{g}{ }^{2} v s$. $\mathrm{P}_{\text {det }}$ is 0.764 and 0.671 , respectively. The median and mean value of the correlation coefficients between $c_{g}{ }^{2}$ and $\mathrm{P}_{\text {ves }}$ are 0.872 and 0.777 , respectively, indicating that $c_{g}{ }^{2}$ shows a stronger correlation with $\mathrm{P}_{\text {ves }}$ than with $\mathrm{P}_{\text {det. }}$. Figure 3 demonstrates that the overall correlation is moderately strong $(r=0.675, \mathrm{P}<0.001)$ between $c_{g}{ }^{2}$ and $\mathrm{P}_{\text {ves }}$ for the aggregate 705 measurements recorded from all patients. There is also a moderately strong overall correlation $(r=0.612, \mathrm{P}<0.001)$ between $c_{g}{ }^{2}$ and $\mathrm{P}_{\mathrm{det}}$.

Urological diagnoses indicated that 22 out of a total 79 patients had non-compliant bladders $\left(<40.0 \mathrm{~mL} / \mathrm{cmH}_{2} \mathrm{O}\right)$, and the rest had compliant bladders. The UBV analysis of compliance was based on the compliance index, as defined in Eq. [2]. Based on this analysis, the resulting box-line plot distributions are presented in Figure $4 A$. The orange square markers refer to the median value with the error bars showing $95 \%$ confidential interval (CI) from the median value. The Student $t$-test found that the group difference is significant $(\mathrm{P}<0.008)$. The ROC curve in Figure $4 B$ demonstrates the performance of the compliance index on differentiating the non-compliant from the compliant bladders. The areas under curve are 0.813 , with $95 \%$ CI ranging from 0.709 to 0.892 . The optimal criterion for the test is that a bladder is considered non-compliant if the compliance index is less than $100 \mathrm{~mL} \cdot \mathrm{s}^{2} \cdot \mathrm{m}^{-2}$, and non-compliant otherwise. The resulting sensitivity and specificity are $86.4 \%$ and $71.9 \%$, respectively. These results suggest that the LWS-related parameter $\left(c_{g}{ }^{2}\right)$ can be used as an alternative indicator to assess bladder compliance with acceptable accuracy.

\section{Discussion}

The goal of this study was to investigate the performance UBV as a diagnostic tool and the Compliance Index ad a new biomarker for differentiating between compliant and non-compliant bladders.

Results of our study demonstrated that the changes in $c_{g}{ }^{2}$ are correlated with those of $\mathrm{P}_{\text {ves }}$ and $\mathrm{P}_{\text {det. }}$. The median and mean values of the correlation coefficient between $c_{g}{ }^{2}$ and $\mathrm{P}_{\text {ves }}$ are 0.872 and 0.777 , which indicate that $c_{g}{ }^{2}$ has a stronger correlation with $\mathrm{P}_{\mathrm{ves}}$ than with $\mathrm{P}_{\text {det }}$. This can be a result of pressure measurement variations. As shown in Figure $2 A$, both $\mathrm{P}_{\text {ves }}$ and $c_{g}^{2}$ are quite stable with the increasing filling volume while the corresponding $\mathrm{P}_{\mathrm{det}}$ varied slightly, which can be the result of measurement error. Further investigations are needed to better understand the correlation between $c_{g}{ }^{2}$ and $\mathrm{P}_{\text {ves }}$. Considering that $c_{g}{ }^{2}$ shows only a moderately strong correlation with the pressure measurements, the above results also suggest that there might be other confounding factors, such as patient-related variabilities, measurement error and experimental factors, 
Table 2 Summary of the statistical analysis of correlation between UDS pressure measurement and Lamb wave group velocity squared ( $\mathrm{c}_{\mathrm{g}}{ }^{2)}$

\begin{tabular}{llc}
\hline Correlation variables & Corr $\left(\mathrm{c}_{\mathrm{g}}{ }^{2}, \mathrm{P}_{\mathrm{det}}\right)$ & Corr $\left(\mathrm{c}_{\mathrm{g}}{ }^{2}, \mathrm{P}_{\text {ves }}\right)$ \\
\hline Median Pearson Corr $(95 \% \mathrm{Cl})$ & $0.764(0.681 \sim 0.850)$ & $0.872(0.826 \sim 0.912)$ \\
Mean Pearson Corr $(95 \% \mathrm{Cl})$ & $0.671(0.597 \sim 0.745)$ & $0.777(0.718 \sim 0.837)$ \\
\hline
\end{tabular}

UDS, urodynamic study; $\mathrm{c}_{\mathrm{g}}{ }^{2}$, Lamb wave group velocity squared; Corr, correlation; Cl, confidence interval; $P_{\text {det }}$, detrusor pressure; $P_{\text {ves }}$, intravesical pressure.

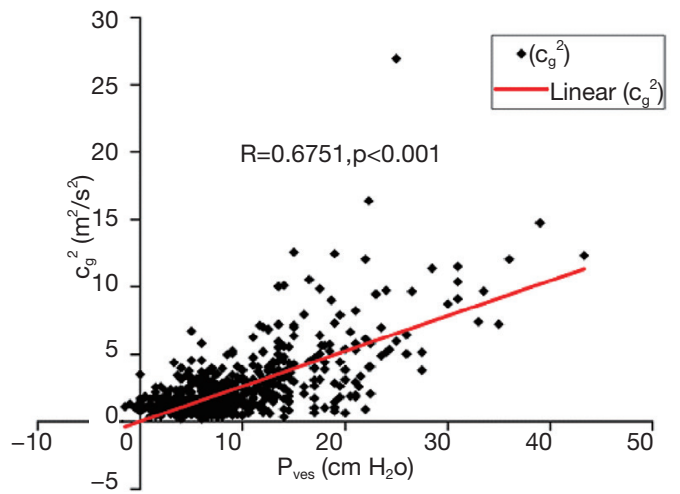

Figure 3 The overall correlation between $c_{g}{ }^{2}$ and Pves for the aggregate 696 measurements obtained from all patients. Multiple measurements were obtained from each patient. Each marker refers to one measurement of a patient.

which may affect the correlation between $c_{g}{ }^{2}$ the two pressure measurements.

Our study also demonstrated that $c_{g}{ }^{2}$ can be used to differentiate between non-compliant and compliant bladders. The compliance index defined based on Eq. [2], showed a significant difference between the two groups. The optimal criterion for the compliance index was found to be $100 \mathrm{~mL} \cdot \mathrm{s}^{2} \cdot \mathrm{m}^{-2}$, and the resulting sensitivity and specificity were $86.4 \%$ and $71.9 \%$, respectively.

It is of interest to compare our results with those of other studies on the use of ultrasound for assessment of bladder functions. Sturm et al. [2017] measured shear wave speed (SWS) at four volume-intervals throughout filling in 23 children and found that SWS of the anterior bladder wall has a significant correlation with $\mathrm{P}_{\text {det }}$, and that SWS was significantly greater in non-compliant than in compliant bladders throughout the filling phase (19). Bavani
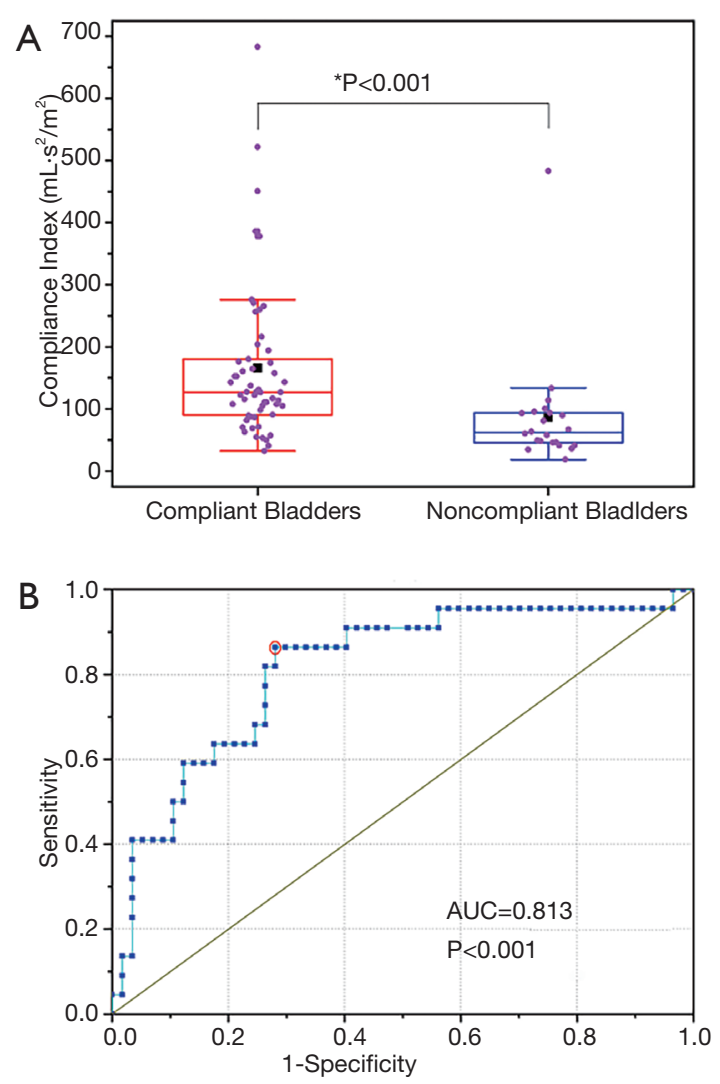

Figure 4 Statistical analysis of the compliance index. (A) The boxand-whisker plots of the compliance index for the compliant and non-compliant bladder groups. The black square refers to the mean of compliance index in a group. Student $t$-test shows there is significant difference between these two groups $(\mathrm{P}<0.008)$; (B) the receiver-operating characteristic (ROC) curve of the compliance index. The area under curve is 0.813 with $95 \% \mathrm{CI}$ ranging from 0.709 to 0.892 . The red circle marker refers to the optimal criterion, where the sensitivity and specificity are $86.4 \%$ and $71.9 \%$, respectively, for the optimal criterion. 
et al. [2019] conducted a study on 30 children with NB and found that SWS of the anterior bladder wall in subjects with neurogenic was significantly higher than children with a normal bladder. Also, in subjects with neurogenic bladder, they found a significant correlation between the mean SWS and the detrusor pressure at the estimated bladder volume based on the aggregate measurements from all 30 patients (20). Our study was made on 79 adult patients and defined a new compliance index, as defined in Eq. [2] that could discriminate between the non-compliant and compliant bladders with acceptable accuracy. The definition of compliance index implies that only one measurement of $c_{g}{ }^{2}$ at the end of the filling phase is sufficient for assessing bladder compliance, which agrees with the conclusion of Bavani et al. Our study is different from other studies mentioned above on several aspects. First, the patient age and population are different. Our study focuses on adult patients and includes a relative larger cohort. Second, our study measured the $c_{g}^{2}$ at every $50 \mathrm{~mL}$ increment throughout the filling and examine the correlation of $c_{g}^{2}$ with $\mathrm{P}_{\text {det }}$ and $\mathrm{P}_{\text {ves }}$ for each individual. Our results demonstrate that $c_{g}{ }^{2}$ is strongly correlated with both $\mathrm{P}_{\text {det }}$ as well as $\mathrm{P}_{\text {ves }}$ in most of the patients, suggesting that $c_{g}{ }^{2}$ can be used as an indicator of bladder noncompliance.

The study presented in this paper provides evidence supporting the potential use of ultrasound for evaluating mechanical properties of the bladder, and specifically for assessing bladder compliance. As a practical note, the method presented here can be employed without bladder catheterization, i.e., without artificially filling the bladder. Since the compliance index defined in Eq. [2] is based on the maximum bladder volume, only a single ultrasound measurement is needed when the patient's bladder is naturally filled with urine. The results of this paper open the way for future application of UBV as a potential noninvasive and low-cost replacement for UDS for certain patient populations.

\section{Conclusions}

A clinical study was performed on 79 adult patients with neurogenic bladder dysfunction to evaluate the performance of UVB technique in the determination of bladder compliance. The results found that the LWS squared, $c_{g}{ }^{2}$ is strongly correlated with both the detrusor and intravesical pressures for most patients in this study. The proposed Compliance Index, which is a biomarker defined based on $c_{g}{ }^{2}$ demonstrated the ability to discriminate the non-compliant and compliant bladders with acceptable accuracy. The results indicate that UBV may be used as a non-invasive method for evaluating the biomechanical changes in the bladder wall, and it may serve as a non-invasive alternative method to the UDS in the assessment of bladder compliance.

\section{Acknowledgments}

The authors would like to thank Dr. Max Denis, Dr. Mahdi Bayat, Dr. Viksit Kumar, Dr. Baehyung Kim, Dr. Rohit Nayak, Mr. Jeremy Webb, and MS. Adriana Gregory for their contribution to data collection. The authors acknowledge David Rosen's assistance in editing the manuscript. The authors also would like to thank Duane Meixner for his help in ultrasound scanning, and Pamela Draayer, Jane Smith, Cynthia Andrist and Barbara Foreman for their assistance in recruiting the patients.

Funding: This work was supported in part by the National Institutes of Health (NIH), [Grant R01DK099231, PI: M.F.]. The NIH did not have any additional role in the study design, data collection and analysis, decision to publish or preparation of the manuscript.

\section{Footnote}

Reporting Checklist: The authors have completed the STARD checklist. Available at http://dx.doi.org/10.21037/atm $-20-6900$

Data Sharing Statement: Available at http://dx.doi. org/10.21037/atm-20-6900

Conflicts of Interest: All authors have completed the ICMJE uniform disclosure form (available at http://dx.doi. org/10.21037/atm-20-6900). MF is an inventor of US patent $9,345,448$ on the technology used in this study. The other authors have no conflicts of interest to declare.

Etbical Statement: The authors are accountable for all aspects of the work in ensuring that questions related to the accuracy or integrity of any part of the work are appropriately investigated and resolved. The study was Health Insurance Portability and Accountability Act Compliant was conducted in accordance with the Declaration of Helsinki (as revised in 2013), and was approved by the Mayo Clinic Institutional Review Board (IRB) (IRB\#: 11-001953). A signed written informed consent with permission for publication was obtained from 
each enrolled patient prior to the study.

Open Access Statement: This is an Open Access article distributed in accordance with the Creative Commons Attribution-NonCommercial-NoDerivs 4.0 International License (CC BY-NC-ND 4.0), which permits the noncommercial replication and distribution of the article with the strict proviso that no changes or edits are made and the original work is properly cited (including links to both the formal publication through the relevant DOI and the license). See: https://creativecommons.org/licenses/by-nc-nd/4.0/.

\section{References}

1. Abrams P, Cardozo L, Fall M, et al. The standardisation of terminology of lower urinary tract function: report from the Standardisation Sub-committee of the International Continence Society. Neurourol Urodyn 2002;21:167-78.

2. Kim AK, Hill WG. Effect of filling rate on cystometric parameters in young and middle aged mice. Bladder 2017;4.

3. Mahfouz W, Al Afraa T, Campeau L, et al. Normal urodynamic parameters in women: part II--invasive urodynamics. Int Urogynecol J 2012;23:269-77.

4. Liao JY, Lin YH, Liang CC, et al. Monitoring bladder compliance using end filling detrusor pressure: Clinical results and related factors. Taiwanese Journal of Obstetrics and Gynecology 2015;54:709-15.

5. Suskind AM, Clemens JQ, Kaufman SR, et al. Patient perceptions of physical and emotional discomfort related to urodynamic testing: a questionnaire-based study in men and women with and without neurologic conditions. Urology 2015;85:547-51.

6. Nóbrega MM, Auge APF, de Toledo LGM, et al. Bacteriuria and urinary tract infection after female urodynamic studies: Risk factors and microbiological analysis. Am J Infect Control 2015;43:1035-9.

7. Nadeem M, Sheikh MI, Sait MS, et al. Is urinary tract infection after urodynamic study predictable? Urol Sci 2017;28:240-2.

8. Nagle AS, Klausner AP, Varghese J, et al. Quantification of bladder wall biomechanics during urodynamics: A methodologic investigation using ultrasound. J Biomech 2017;61:232-41.

9. Nagle AS, Bernardo RJ, Varghese J, et al. Comparison of 2D and 3D ultrasound methods to measure serial bladder volumes during filling: Steps toward development of noninvasive ultrasound urodynamics. Bladder 2018;5:e32.

10. Sigrist RMS, Liau J, Kaffas AE, et al. Ultrasound
Elastography: Review of Techniques and Clinical Applications. Theranostics 2017;7:1303-29.

11. Nenadic I, Mynderse L, Husmann D, et al. Noninvasive Evaluation of Bladder Wall Mechanical Properties as a Function of Filling Volume: Potential Application in Bladder Compliance Assessment. PLoS One 2016;11:e0157818.

12. Bayat M, Kumar V, Denis M, et al. Correlation of ultrasound bladder vibrometry assessment of bladder compliance with urodynamic study results. PLoS One 2017;12:e0179598.

13. Nenadic IZ, Qiang B, Urban MW, et al. Ultrasound bladder vibrometry method for measuring viscoelasticity of the bladder wall. Phys Med Biol 2013;58:2675-95.

14. Akkus Z, Kim BH, Nayak R, et al. Fully Automated Segmentation of Bladder Sac and Measurement of Detrusor Wall Thickness from Transabdominal Ultrasound Images. Sensors 2020;20:4175.

15. Sturm RM, Yerkes EB, Nicholas JL, et al. Ultrasound shear wave elastography: a novel method to evaluate bladder pressure. J Urol 2017;198:422-9.

16. Tanter M, Fink M. Ultrafast imaging in biomedical ultrasound. IEEE Trans Ultrason Ferroelectr Freq Control 2014;61:102-19.

17. Loupas T, Powers JT, Gill RW. An Axial Velocity Estimator for Ultrasound Blood-Flow Imaging, Based on a Full Evaluation of the Doppler Equation by Means of a 2-Dimensional Autocorrelation Approach. IEEE Trans Ultrason Ferroelectr Freq Control 1995;42:672-88.

18. Toppercer A, Tetreault JP. Compliance of the bladder: an attempt to establish normal values. Urology 1979;14:204-5.

19. Sturm Renea M, Yerkes Elizabeth B, Nicholas Jennifer L, et al. Ultrasound Shear Wave Elastography: A Novel Method to Evaluate Bladder Pressure. J Urol 2017;198:422-9.

20. Bavani AG, Hanafi MG, Sarkarian M. An investigation into the sensitivity of shear wave ultrasound elastography to measure the anterior bladder wall pressure in patients with neurogenic bladder. J Family Med Prim Care 2019;8:1342-6.

Cite this article as: Zhang X, Husmann DA, Mynderse LA, Alizad A, Fatemi M. Non-invasive assessment of urinary bladder compliance using ultrasound: first validation study based on clinical urodynamic study. Ann Transl Med 2021;9(7):547. doi: 10.21037/atm-20-6900 